\title{
Singular Limit of the Rotational Compressible Magnetohydrodynamic Flows
}

\author{
Young-Sam Kwon \\ Department of Mathematics, Dong-A University, Busan 604-714, Republic of Korea \\ Correspondence should be addressed to Young-Sam Kwon; ykwon@dau.ac.kr
}

Received 4 April 2017; Revised 24 May 2017; Accepted 6 June 2017; Published 25 July 2017

Academic Editor: Luigi C. Berselli

Copyright (C) 2017 Young-Sam Kwon. This is an open access article distributed under the Creative Commons Attribution License, which permits unrestricted use, distribution, and reproduction in any medium, provided the original work is properly cited.

\begin{abstract}
We consider the compressible models of magnetohydrodynamic flows giving rise to a variety of mathematical problems in many areas. We derive a rigorous quasi-geostrophic equation governed by magnetic field from the stratified flows of the rotational compressible magnetohydrodynamic flows with the well-prepared initial data and the tool of proof is based on the relative entropy. Furthermore, the convergence rates are obtained.
\end{abstract}

\section{Introduction}

Magnetohydrodynamic flows arise in science and engineering in a variety of practical applications such as in plasma confinement, liquid-metal cooling of nuclear reactors, and electromagnetic casting. The fundamental concept behind MHD flows is that magnetic fields can induce currents in a moving conductive fluid, which in turn polarizes the fluid and reciprocally changes the magnetic field itself. The set of equations that describe MHD flows are a combination of the Navier-Stokes equations of fluid dynamics and Maxwell's equations of electromagnetism. These differential equations must be solved simultaneously, either analytically or numerically. Here we consider the viscous rotational compressible magnetohydrodynamic flows in the 2-dimensional whole space $\Omega:=\mathbb{R}^{2}$ :

$$
\begin{aligned}
& \partial_{t} \varrho_{\varepsilon}+\operatorname{div}\left(\varrho_{\varepsilon} \mathbf{u}_{\varepsilon}\right)=0, \\
& \partial_{t}\left(\varrho_{\epsilon} \mathbf{u}_{\epsilon}\right)+\operatorname{div}\left(\varrho_{\epsilon} \mathbf{u}_{\epsilon} \otimes \mathbf{u}_{\epsilon}\right)+\frac{1}{\varepsilon^{2} \gamma} \nabla \varrho_{\epsilon}^{\gamma}+\frac{1}{\varepsilon} \varrho_{\epsilon} \mathbf{u}_{\epsilon}^{\perp} \\
& =\mu_{\varepsilon} \Delta \mathbf{u}_{\epsilon}+\left(\mu_{\varepsilon}+\lambda_{\varepsilon}\right) \nabla\left(\operatorname{div} \mathbf{u}_{\epsilon}\right)+\left(\mathbf{B}_{\varepsilon} \cdot \nabla\right) \mathbf{B}_{\varepsilon} \\
& -\frac{1}{2} \nabla\left|\mathbf{B}_{\varepsilon}\right|^{2}+\frac{1}{\varepsilon} \varrho_{\epsilon} \nabla G, \\
& \partial_{t} \mathbf{B}_{\varepsilon}+\left(\operatorname{div} \mathbf{u}_{\epsilon}\right) \mathbf{B}_{\varepsilon}+\left(\mathbf{u}_{\epsilon} \cdot \nabla\right) \mathbf{B}_{\varepsilon}-\left(\mathbf{B}_{\varepsilon} \cdot \nabla\right) \mathbf{u}_{\epsilon}=v_{\varepsilon} \Delta \mathbf{B}_{\varepsilon},
\end{aligned}
$$

where $\mathbf{u}_{\epsilon}$ is the vector field, $\varrho_{\epsilon}$ is the density, $\mathbf{B}_{\varepsilon}$ is the magnetic field, $\gamma>3 / 2$, and $G \in C_{c}^{3}(\Omega)$, and we also assume that

$$
\begin{aligned}
& \mu_{\varepsilon}=\varepsilon^{\theta} \\
& v_{\varepsilon}=\varepsilon^{\sigma} \\
& \lambda_{\varepsilon} \longrightarrow 0,
\end{aligned}
$$

with $\theta, \sigma>0$, as $\varepsilon$ tends to 0 .

We first notice that the global-in-time existence solutions for systems ((1)-(3)), supplemented with physically relevant constitutive relations, has been studied by Hu and Wang [1].

It should be pointed out that the incompressible inviscid limit problems to the compressible Navier-Stokes equations and related models are very interesting. For the case without rotational force, Masmoudi [2] proved the convergence of the weak solution of isentropic Navier-Stokes equations to the strong solution of the incompressible Euler equations in the 2-dimensional whole space $\mathbb{R}^{2}$ and the space case by applying the related entropy method. Later, his result was extended to the isentropic compressible magnetohydrodynamic equations [3, 4]. Feireisl and Novotný [5] studied the inviscid incompressible limit to the full Navier-Stokes-Fourier system in the whole space.

In this paper, we derive a rigorous quasi-geostrophic equation from the stratified flow of rotational compressible magnetohydrodynamic flows ((1)-(3)) on the 2-dimensional 
whole space with the well-prepared initial data. Our contribution of this paper is physically to derive a rigorous quasi-geostrophic equation from the stratified flows of the rotational compressible magnetohydrodynamic equations based on the relative entropy method. Recently, Feireisl and Novotný [6] have studied the asymptotic limit for the models with a rotational term originating from a Coriolis force with the mild stratification and with the well-prepared initial data. This result is based on their paper, but it is more a developed version than their result because we derive a quasi-geostrophic equation from a global weak solution of compressible MHD flows.

Let the density $\widetilde{\varrho}$ be the solution of the static problem

$$
\nabla \widetilde{\varrho}_{\varepsilon}^{\gamma}=\gamma \varepsilon \widetilde{\varrho}_{\varepsilon} \nabla G, \quad \widetilde{\varrho}_{\varepsilon}(x) \longrightarrow 1 \text { as }|x| \longrightarrow \infty,
$$

where we have

$$
\begin{aligned}
\left|\widetilde{\varrho}_{\varepsilon}(x)-1\right| & \leq \varepsilon|G(x)|, \\
\left|\nabla \widetilde{\varrho}_{\varepsilon}(x)\right| & \leq \varepsilon|\nabla G(x)|,
\end{aligned}
$$

$$
\tilde{\varrho}_{\varepsilon} \in C^{3}(\Omega), x \in \Omega .
$$

Assume that the initial data have the following property at infinity:

$$
\begin{aligned}
& \varrho_{\epsilon}(x) \longrightarrow 1, \\
& \mathbf{u}_{\epsilon}(x) \longrightarrow 0, \\
& \mathbf{B}_{\varepsilon}(x) \longrightarrow 0 \\
& \quad \text { as }|x| \longrightarrow \infty .
\end{aligned}
$$

Formally, we will investigate the limit

$$
\begin{aligned}
\frac{\varrho_{\epsilon}-\widetilde{\varrho}_{\varepsilon}}{\varepsilon} & \longrightarrow q, \\
\sqrt{\varrho_{\epsilon}} \mathbf{u}_{\epsilon} & \longrightarrow \mathbf{v}, \\
\mathbf{B}_{\varepsilon} & \longrightarrow \mathbf{B},
\end{aligned}
$$

as $\epsilon$ tends to 0 in the suitable sense such that the given limits $(q, B)$ represent the unique local smooth strong solution of the following system on $[0, T]$ : for $q_{0} \in H^{k+1}(\Omega)$ and $\mathbf{B}_{0} \in$ $H^{k}(\Omega), k \geq 3$,

$$
\begin{aligned}
& \mathbf{v}=\nabla^{\perp} q, \\
& \partial_{t}(\Delta q-q)+\left(\nabla^{\perp} q \cdot \nabla\right) \Delta q+\nabla^{\perp} q \cdot \nabla G \\
& \quad=\operatorname{div}^{\perp}[(\mathbf{B} \cdot \nabla) \mathbf{B}], \\
& q(0, \cdot)=q_{0} \\
& \partial_{t} \mathbf{B}+\left(\nabla^{\perp} q \cdot \nabla\right) \mathbf{B}-(\mathbf{B} \cdot \nabla) \nabla^{\perp} q=0, \\
& \mathbf{B}(0, \cdot)=\mathbf{B}_{0},
\end{aligned}
$$

where the notations are defined as follows:

$$
\begin{aligned}
\mathbf{v}^{\perp} & =\left(-v_{2}, v_{1}\right), \\
\nabla^{\perp} g & =\left(-\frac{\partial g}{\partial x_{2}}, \frac{\partial g}{\partial x_{1}}\right), \\
\operatorname{div}^{\perp} \mathbf{v} & =-\frac{\partial v_{1}}{\partial x_{2}}+\frac{\partial v_{2}}{\partial x_{1}} .
\end{aligned}
$$

Note that the existence of global strong solution of system (9) can be proven with the same method of [7].

Theorem 1. Let $\Omega$ be the 2-dimensional whole space $\mathbb{R}^{2}$. For the given initial $q_{0} \in H^{k+1}(\Omega)$ and $\mathbf{B}_{0} \in H^{k}(\Omega), k \geq 3$, there is $T>0$ such that system (9) has the unique local smooth solution $(q, \mathbf{B})$ on $[0, T]$, verifying the following regularity:

$$
\begin{aligned}
q \in & C\left([0, T] ; H^{k+1}(\Omega ; \mathbb{R})\right) \\
& \cap C^{1}\left([0, T] ; H^{k}(\Omega ; \mathbb{R})\right), \\
\mathbf{B} \in & C\left([0, T] ; H^{k}\left(\Omega ; \mathbb{R}^{2}\right)\right) \\
& \cap C^{1}\left([0, T] ; H^{k-1}\left(\Omega ; \mathbb{R}^{2}\right)\right) .
\end{aligned}
$$

The outline of this article is as follows. In Section 2, we present the rigorous result for (8) and (9). In Section 3, we derive a rigorous proof of the rotational compressible magnetohydrodynamic flows ((1)-(3)).

Definition 2. We say that a quantity $\{\varrho, \mathbf{u}, \mathbf{B}\}$ is a weak solution of the magnetohydrodynamic (MHD) flows ((1)-(3)) supplemented with the initial data $\left\{\varrho_{0}, \mathbf{u}_{0}, \mathbf{B}_{0}\right\}$ provided that the following hold:

(i) The density $\varrho$ is a nonnegative function, where $\varrho$ $1 \in L^{\infty}\left(0, T ;\left(L^{\gamma}+L^{2}\right)(\Omega)\right)$, the velocity field $\mathbf{u} \in$ $L^{2}\left(0, T ; W^{1,2}\left(\Omega ; \mathbb{R}^{2}\right)\right), \varrho|\mathbf{u}|^{2} \in L^{\infty}\left(0, T ; L^{1}(\Omega)\right)$, and $\mathbf{u}$ represents a renormalized solution of equation (1) on $(0, T) \times \Omega$; that is, the integral identity

$$
\begin{aligned}
& \int_{\Omega}(\varrho+b(\varrho)) \varphi(T, \cdot) \mathrm{d} x-\int_{\Omega}\left(\varrho_{0}+b\left(\varrho_{0}\right)\right) \varphi(0, \cdot) \mathrm{d} x \\
& \quad=\int_{0}^{T} \int_{\Omega}\left[(\varrho+b(\varrho)) \partial_{t} \varphi+(\varrho+b(\varrho)) \mathbf{u} \cdot \nabla \varphi\right. \\
& \left.\quad+\left(b(\varrho)-b^{\prime}(\varrho) \varrho\right) \operatorname{div} \mathbf{u} \varphi\right] \mathrm{d} x \mathrm{~d} t
\end{aligned}
$$

holds for any test function $\varphi \in \mathscr{D}([0, T) \times \Omega)$ and any $b$ such that

$$
b \in C^{1}[0, \infty), \quad b^{\prime}(r)=0 \text { whenever } r \geq r_{b} .
$$


(ii) The balance of momentum holds in distributional sense; namely,

$$
\begin{aligned}
\int_{\Omega} & \mathbf{u} \cdot \vec{\varphi}(T, \cdot) \mathrm{d} x-\int_{\Omega}(\varrho \mathbf{u})_{0} \cdot \vec{\varphi}(0, \cdot) \mathrm{d} x \\
& =\int_{0}^{T} \int_{\Omega}\left(\varrho \mathbf{u} \cdot \partial_{t} \vec{\varphi}+\varrho \mathbf{u} \otimes \mathbf{u}: \nabla \vec{\varphi}+\frac{1}{\epsilon^{2} \gamma} \varrho^{\gamma} \operatorname{div} \vec{\varphi}\right. \\
& \left.+\left[(\mathbf{B} \cdot \nabla) \mathbf{B}-\frac{1}{2} \nabla|\mathbf{B}|^{2}\right] \cdot \vec{\varphi}\right) \mathrm{d} x \mathrm{~d} t \\
& -\int_{0}^{T} \int_{\Omega}\left(\frac{1}{\varepsilon} \varrho \mathbf{u}^{\perp} \cdot \vec{\varphi}-\mu_{\varepsilon} \nabla \mathbf{u}: \nabla \vec{\varphi}\right. \\
- & \left.\left(\mu_{\varepsilon}+\lambda_{\varepsilon}\right) \operatorname{div} \mathbf{u} \operatorname{div} \vec{\varphi}+\frac{1}{\varepsilon} \varrho \nabla G \cdot \vec{\varphi}\right) \mathrm{d} x \mathrm{~d} t
\end{aligned}
$$

for any test function $\vec{\varphi} \in \mathscr{D}\left([0, T) \times \Omega ; \mathbb{R}^{2}\right)$.

(iii) The total energy of the system holds:

$$
\begin{aligned}
& \int_{\Omega}\left(\frac{1}{2} \varrho|\mathbf{u}|^{2}+\frac{1}{2}|\mathbf{B}|^{2}\right. \\
& \left.+\frac{1}{\gamma(\gamma-1) \varepsilon^{2}}\left(\varrho^{\gamma}-\widetilde{\varrho}_{\varepsilon}^{\gamma}-\gamma \widetilde{\varrho}_{\varepsilon}^{\gamma-1}\left(\varrho-\widetilde{\varrho}_{\varepsilon}\right)\right)\right) \\
& \cdot(t, \cdot) \mathrm{d} x+\int_{0}^{t} \int_{\Omega} \mu_{\varepsilon}|\nabla \mathbf{u}|^{2}+\left(\mu_{\varepsilon}+\lambda_{\varepsilon}\right)(\operatorname{div} \mathbf{u})^{2} \\
& +v_{\varepsilon}|\nabla \mathbf{B}|^{2} \mathrm{~d} x \mathrm{~d} t \leq E_{0, \epsilon}
\end{aligned}
$$

holds for a.e. $t \in(0, T)$, where

$$
\begin{aligned}
E_{0, \epsilon} & =\int_{\Omega}\left(\frac{1}{2} \varrho_{0}\left|\mathbf{u}_{0}\right|^{2}+\frac{1}{2}\left|\mathbf{B}_{0}\right|^{2}\right. \\
& \left.+\frac{1}{\gamma(\gamma-1) \varepsilon^{2}}\left(\varrho_{0}^{\gamma}-\widetilde{\varrho}_{\varepsilon}^{\gamma}-\gamma \widetilde{\varrho}_{\varepsilon}^{\gamma-1}\left(\varrho_{0}-\widetilde{\varrho}_{\varepsilon}\right)\right)\right) \mathrm{d} x .
\end{aligned}
$$

(iv) The Maxwell equation (3) with $\operatorname{div} \mathbf{B}=0$ and the regularity $\mathbf{B} \in L^{2}\left(0, T ; W^{1,2}(\Omega)\right) \cap L^{\infty}\left(0, T ; L^{2}(\Omega)\right)$ verifies

$$
\begin{aligned}
& \int_{\Omega} \mathbf{B} \cdot \vec{\varphi}(T, \cdot) \mathrm{d} x-\int_{\Omega}(\mathbf{B})_{0} \cdot \vec{\varphi}(0, \cdot) \mathrm{d} x=\int_{0}^{T} \int_{\Omega} \mathbf{B} \\
& \cdot \partial_{t} \vec{\varphi} \mathrm{d} x \mathrm{~d} t-\int_{0}^{T} \int_{\Omega}((\mathbf{u} \times \mathbf{B}) \cdot(\nabla \times \vec{\varphi}) \\
& -(\nu \nabla \times \mathbf{B}) \cdot(\nabla \times \vec{\varphi})) \mathrm{d} x \mathrm{~d} t, \\
& \quad \text { for all } \vec{\varphi} \in \mathscr{D}\left([0, T) \times \Omega ; \mathbb{R}^{2}\right) .
\end{aligned}
$$

\section{Main Results}

In this section, we introduce the main results.

Theorem 3. Let $\Omega=\mathbb{R}^{2}$ be the 2-dimensional whole space and let $\left(\varrho_{\epsilon}, \mathbf{u}_{\epsilon}, \mathbf{B}_{\varepsilon}\right)$ be a weak solution to ((1)-(3)) in the sense of Definition 2, verifying viscosity (4) with $0<\theta+\sigma<2$ and the initial data:

$$
\begin{array}{r}
\left\|q_{0, \varepsilon}-q_{0}\right\|_{L^{2}(\Omega)}^{2} \leq C \varepsilon, \\
\left\|H_{\varepsilon}\left(q_{0, \varepsilon}\right)-q_{0}\right\|_{L^{2}(\Omega)}^{2} \leq C \varepsilon, \\
\left\|\sqrt{\varrho_{0, \varepsilon}} \mathbf{u}_{0, \varepsilon}-\nabla^{\perp} q_{0}\right\|_{L^{2}(\Omega)}^{2} \leq C \varepsilon, \\
\left\|\mathbf{B}_{0, \varepsilon}-\mathbf{B}_{0}\right\|_{L^{2}(\Omega)}^{2} \leq C \varepsilon,
\end{array}
$$

where

$$
\begin{gathered}
\varrho_{0, \varepsilon}=\widetilde{\varrho}_{\varepsilon}+\varepsilon q_{0, \varepsilon}, \\
q_{0, \varepsilon} \in L^{2} \cap L^{\infty}(\Omega), q_{0} \in H^{k+1}(\Omega), \\
\mathbf{v}_{0}, \mathbf{B}_{0} \in H^{k}\left(\Omega ; \mathbb{R}^{3}\right),
\end{gathered}
$$

for $k \geq 3$. Then, one has

$$
\begin{gathered}
\left\|\frac{\varrho_{\epsilon}-\widetilde{\varrho}_{\varepsilon}}{\varepsilon}-q\right\|_{L^{\infty}\left(0, T ; L^{1}(K)\right)} \leq C(K) \varepsilon^{\kappa} \\
\left\|\sqrt{\varrho_{\epsilon}} \mathbf{u}_{\varepsilon}-\mathbf{v}\right\|_{L^{\infty}\left(0, T ; L^{2}\left(\Omega ; \mathbb{R}^{2}\right)\right)}^{2} \leq C \varepsilon^{\mathcal{K}} \\
\left\|\mathbf{B}_{\varepsilon}-\mathbf{B}\right\|_{L^{\infty}\left(0, T ; L^{2}(\Omega)\right)}^{2} \leq C \varepsilon^{\mathcal{K}}
\end{gathered}
$$

for sufficiently small $0<\varepsilon<1$, any $T<T_{*}$, and any compact $K \subset \subset \mathbb{R}^{2}$ such that $(q, \mathbf{B})$ verifies (9). Furthermore, the numbers $\kappa$ are defined by

$$
\kappa=\min \left\{1, \frac{2}{\gamma}, 1-\frac{\theta+\sigma}{2}, \frac{5}{3}-\frac{\theta}{2}, \theta, \sigma\right\} .
$$

\section{Proof of Theorem 3}

In this section, we are going to give the rigorous proof of Theorem 3.

Step 1. In this part, we are going to derive some estimates on the sequence $\left\{\varrho_{\epsilon}, \mathbf{u}_{\epsilon}, \mathbf{B}_{\varepsilon}\right\}_{\epsilon>0}$.

From the energy inequality (16), we obtain

$$
\begin{aligned}
\operatorname{ess} \sup _{t \in(0, T)}\left\|\sqrt{\varrho_{\epsilon}} \mathbf{u}_{\epsilon}(t)\right\|_{L^{2}(\Omega)} & \leq C, \\
\operatorname{ess} \sup _{t \in(0, T)}\left\|\varrho_{\epsilon}^{\gamma}-\widetilde{\varrho}_{\varepsilon}^{\gamma}-\gamma \widetilde{\varrho}_{\varepsilon}^{\gamma-1}\left(\varrho_{\epsilon}-\widetilde{\varrho}_{\varepsilon}\right)\right\|_{L^{1}(\Omega)} & \leq \epsilon^{2} C, \\
\left\|\nabla \mathbf{u}_{\epsilon}\right\|_{L^{2}((0, T) \times \Omega)} & \leq C \varepsilon^{-\theta / 2}, \\
\operatorname{ess} \sup _{t \in(0, T)}\left\|\mathbf{B}_{\varepsilon}(t)\right\|_{L^{2}(\Omega)} & \leq C, \\
\left\|\nabla \mathbf{B}_{\varepsilon}\right\|_{L^{2}((0, T) \times \Omega)} & \leq C \varepsilon^{-\sigma / 2} .
\end{aligned}
$$


We consider the properties of convex function:

$$
\begin{aligned}
& \varrho_{\epsilon}^{\gamma}-\widetilde{\varrho}_{\varepsilon}^{\gamma}-\gamma\left(\varrho_{\epsilon}-\widetilde{\varrho}_{\varepsilon}\right) \geq C\left|\varrho_{\epsilon}-\widetilde{\varrho}_{\varepsilon}\right|^{2} \quad \text { if } \gamma \geq 2, \\
& \varrho_{\epsilon}^{\gamma}-\widetilde{\varrho}_{\varepsilon}^{\gamma}-\gamma\left(\varrho_{\epsilon}-\widetilde{\varrho}_{\varepsilon}\right) \geq C\left|\varrho_{\epsilon}-\widetilde{\varrho}_{\varepsilon}\right|^{2} \quad \text { if } \gamma<2, x \leq R, \\
& \varrho_{\epsilon}^{\gamma}-\widetilde{\varrho}_{\varepsilon}^{\gamma}-\gamma\left(\varrho_{\epsilon}-\widetilde{\varrho}_{\varepsilon}\right) \geq C\left|\varrho_{\epsilon}-\widetilde{\varrho}_{\varepsilon}\right|^{\gamma} \quad \text { if } \gamma<2, x \geq R, \\
& \varrho_{\epsilon}^{\gamma}-\widetilde{\varrho}_{\varepsilon}^{\gamma}-\gamma\left(\varrho_{\epsilon}-\widetilde{\varrho}_{\varepsilon}\right) \geq C(\delta)\left|\varrho_{\epsilon}-\widetilde{\varrho}_{\varepsilon}\right|^{\gamma} \\
& \text { if }\left|\varrho_{\epsilon}-\widetilde{\varrho}_{\varepsilon}\right|>\delta>0,
\end{aligned}
$$

where we can see these properties in [8]. Following (28) and (29) together with (25), we get

$$
\text { ess } \sup _{t \in(0, T)}\left\|\left[\frac{\varrho_{\epsilon}-\widetilde{\varrho}_{\varepsilon}}{\epsilon} 1_{\left|\varrho_{e}-\widetilde{\varrho}_{\varepsilon}\right| \leq 1 / 2}\right]\right\|_{L^{2}(\Omega)} \leq C .
$$

Using (31), we also derive

$$
\text { ess } \sup _{t \in(0, T)} \int_{\Omega}\left[\frac{\left|\varrho_{\epsilon}-\widetilde{\varrho}_{\varepsilon}\right|^{\gamma}}{\epsilon^{2}} 1_{\left|\varrho_{e}-\tilde{\varrho}\right| \geq 1 / 2}\right] \mathrm{d} x \leq C,
$$

which gives

$$
\text { ess } \sup _{t \in(0, T)} \int_{\Omega}\left[1+\varrho_{\epsilon}^{\gamma}\right] 1_{\left|\varrho_{e}-\widetilde{\varrho}_{\varepsilon}\right| \geq 1 / 2} \mathrm{~d} x \leq \varepsilon^{2} C .
$$

Note that

$$
\left\|\sqrt{\varrho_{\epsilon}}-\sqrt{\widetilde{\varrho}_{\varepsilon}}\right\|_{L^{\infty}\left(0, T ; L^{2}(\Omega)\right)} \leq \varepsilon C,
$$

while using (28)-(31) implies that

$$
\begin{aligned}
\int_{\Omega}\left|\sqrt{\varrho_{\varepsilon}}-\sqrt{\widetilde{\varrho}_{\varepsilon}}\right|^{2} \mathrm{~d} x= & \int_{\left|\varrho_{\varepsilon}-\widetilde{\varrho}\right| \leq 1 / 2}\left|\sqrt{\varrho_{\epsilon}}-\sqrt{\widetilde{\varrho}_{\varepsilon}}\right|^{2} \mathrm{~d} x \\
& +\int_{\left|\varrho_{e}-\widetilde{\varrho}\right| \geq 1 / 2}\left|\sqrt{\varrho_{\epsilon}}-\sqrt{\widetilde{\varrho}_{\varepsilon}}\right|^{2} \mathrm{~d} x \\
\leq & C \int_{\left|\varrho_{\epsilon}-\widetilde{\varrho}\right| \leq 1 / 2}\left|\varrho_{\epsilon}-\widetilde{\varrho}_{\varepsilon}\right|^{2} \mathrm{~d} x \\
& +C \int_{\left|\varrho_{\epsilon}-\widetilde{\varrho}\right| \geq 1 / 2}\left|\varrho_{\epsilon}-\widetilde{\varrho}_{\varepsilon}\right|^{\gamma} \mathrm{d} x \\
\leq & \varepsilon^{2} C .
\end{aligned}
$$

The Sobolev embedding also gives

$$
\left\|\mathbf{u}_{\epsilon}\right\|_{L^{2}}^{2} \leq C+C \varepsilon^{4 / 3}\left\|\nabla \mathbf{u}_{\epsilon}\right\|_{L^{2}}^{2},
$$

and the proof is provided in [9].

Step 2. We introduce the relative entropy in the version of the magnetohydrodynamic flows. Let us set

$$
\begin{aligned}
h(\varrho) & =\frac{1}{\gamma(\gamma-1)}\left(\varrho^{\gamma}-\widetilde{\varrho}_{\varepsilon}^{\gamma}-\gamma \widetilde{\varrho}_{\varepsilon}^{\gamma-1}\left(\varrho-\widetilde{\varrho}_{\varepsilon}\right)\right), \\
H_{\varepsilon}\left(q_{\varepsilon}\right) & =\frac{\sqrt{2}}{\varepsilon} \operatorname{sign}\left(q_{\varepsilon}\right) \sqrt{h\left(\widetilde{\varrho}_{\varepsilon}+\varepsilon q_{\varepsilon}\right)}, \\
\varrho_{\varepsilon} & =\widetilde{\varrho}_{\varepsilon}+\varepsilon q_{\varepsilon},
\end{aligned}
$$

where

$$
\operatorname{sign}\left(q_{\varepsilon}\right)= \begin{cases}1, & \text { if } \operatorname{sign}\left(q_{\varepsilon}\right)>0, \\ 0, & \text { if } \operatorname{sign}\left(q_{\varepsilon}\right)=0, \\ -1, & \text { if } \operatorname{sign}\left(q_{\varepsilon}\right)<0 .\end{cases}
$$

We define the relative entropy:

$$
\begin{aligned}
& \mathscr{E}_{\varepsilon}(\tau) \\
& =\frac{1}{2} \int_{\Omega}\left(\varrho_{\epsilon}\left|\mathbf{u}_{\epsilon}-\mathbf{v}\right|^{2}+\left|H_{\varepsilon}\left(q_{\varepsilon}\right)-q\right|^{2}+\left|\mathbf{B}_{\varepsilon}-\mathbf{B}\right|^{2}\right) \mathrm{d} x,
\end{aligned}
$$

where we put $\mathbf{v}:=\nabla^{\perp} q$. Adapting $\mathbf{v}$ as a test function to the moment equation (2) provides

$$
\begin{aligned}
& -\int_{\Omega}\left(\varrho_{\epsilon} \mathbf{u}_{\epsilon} \cdot \mathbf{v}\right)(\tau) \mathrm{d} x=-\int_{\Omega}\left(\varrho_{0, \varepsilon} \mathbf{u}_{0, \varepsilon}\right) \cdot \mathbf{v}_{0} \mathrm{~d} x \\
& +\int_{0}^{\tau} \int_{\Omega} \frac{1}{\varepsilon} \varrho_{\epsilon} \mathbf{u}_{\epsilon}^{\perp} \cdot \mathbf{v} \mathrm{d} x \mathrm{~d} t-\int_{0}^{\tau} \int_{\Omega}\left[\varrho_{\epsilon} \mathbf{u}_{\epsilon} \otimes \mathbf{u}_{\epsilon}: \nabla \mathbf{v}\right. \\
& \left.+\left(\mathbf{B}_{\varepsilon} \cdot \nabla\right) \mathbf{B}_{\varepsilon} \cdot \mathbf{v}-\mu_{\varepsilon} \nabla \mathbf{u}_{\epsilon}: \nabla \mathbf{v}\right] \mathrm{d} x \mathrm{~d} t \\
& -\int_{0}^{\tau} \int_{\Omega} \varrho_{\epsilon} \mathbf{u}_{\epsilon} \cdot \partial_{t} \mathbf{v} \mathrm{d} x \mathrm{~d} t-\frac{1}{\varepsilon} \int_{0}^{\tau} \int_{\Omega} \varrho_{\epsilon} \nabla G \\
& \quad \cdot \mathbf{v} \mathrm{d} x \mathrm{~d} t .
\end{aligned}
$$

We also use $q$ as a test function to the continuity equation (1) to deduce that

$$
\begin{aligned}
-\int_{\Omega} q_{\varepsilon} q \mathrm{~d} x= & -\int_{\Omega} q_{0, \varepsilon} q_{0} \mathrm{~d} x-\int_{0}^{\tau} \int_{\Omega} q_{\varepsilon} \partial_{t} q \mathrm{~d} x \mathrm{~d} t \\
& -\int_{0}^{\tau} \int_{\Omega} \frac{1}{\varepsilon} \varrho_{\epsilon} \mathbf{u}_{\epsilon} \cdot \nabla q \mathrm{~d} x \mathrm{~d} t .
\end{aligned}
$$

To compute the relative entropy, we also use $\mathbf{B}$ as a test function to the magnetic field equation (3) and insert (9), which yields

$$
\begin{aligned}
- & \int_{\Omega}\left(\mathbf{B}_{\varepsilon} \cdot \mathbf{B}\right)(\tau) \mathrm{d} x=-\int_{\Omega} \mathbf{B}_{0, \varepsilon} \cdot \mathbf{B}_{0} \mathrm{~d} x+\int_{0}^{\tau} \int_{\Omega} \mathbf{B}_{\varepsilon} \\
\cdot & {[(\mathbf{v} \cdot \nabla) \mathbf{B}-(\mathbf{B} \cdot \nabla) \mathbf{v}] \mathrm{d} x \mathrm{~d} t } \\
+ & \int_{0}^{\tau} \int_{\Omega}\left[\left(\operatorname{div} \mathbf{u}_{\epsilon}\right) \mathbf{B}_{\varepsilon}+\left(\mathbf{u}_{\epsilon} \cdot \nabla\right) \mathbf{B}_{\varepsilon}-\left(\mathbf{B}_{\varepsilon} \cdot \nabla\right) \mathbf{u}_{\epsilon}\right] \\
\cdot & \mathbf{B} \mathrm{d} x \mathrm{~d} t+\int_{0}^{\tau} \int_{\Omega} \nu_{\varepsilon} \nabla \mathbf{B}_{\varepsilon}: \nabla \mathbf{B} \mathrm{d} x \mathrm{~d} t .
\end{aligned}
$$

Adding (16), (41), (42), and (43) derives the following inequality:

$$
\begin{aligned}
& \mathscr{E}_{\varepsilon}(\tau)+\int_{0}^{\tau} \int_{\Omega}\left(\mu_{\varepsilon}\left|\nabla \mathbf{u}_{\epsilon}\right|^{2}+\left(\mu_{\varepsilon}+\lambda_{\varepsilon}\right)\left|\operatorname{div} \mathbf{u}_{\epsilon}\right|^{2}\right. \\
& \left.+v_{\varepsilon}\left|\nabla \mathbf{B}_{\varepsilon}\right|^{2}\right) \mathrm{d} x \mathrm{~d} t \leq \sum_{j=1}^{7} A_{j}
\end{aligned}
$$


where

$$
\begin{aligned}
A_{1} & =\mathscr{C}_{\varepsilon}(0)+\int_{\Omega} q_{0}\left(H_{\varepsilon}\left(q_{0, \varepsilon}\right)-q_{0, \varepsilon}\right) \mathrm{d} x-\frac{1}{2} \int_{\Omega}\left(\varrho_{0, \varepsilon}\right. \\
& \left.-\widetilde{\varrho}_{\varepsilon}\right)\left|\nabla^{\perp} q_{0}\right|^{2} \mathrm{~d} x \\
A_{2} & =\frac{1}{2} \int_{\Omega}\left(\varrho_{\epsilon}-\widetilde{\varrho}_{\varepsilon}\right)\left|\nabla^{\perp} q\right|^{2} \mathrm{~d} x-\int_{\Omega} q\left(H_{\varepsilon}\left(q_{\varepsilon}\right)\right. \\
& \left.-q_{\varepsilon}\right) \mathrm{d} x \\
A_{3} & =\frac{1}{2} \int_{\Omega}\left(\widetilde{\varrho}_{\varepsilon}\left|\nabla^{\perp} q\right|^{2}-\widetilde{\varrho}_{\varepsilon}\left|\nabla^{\perp} q_{0}\right|^{2}+q^{2}-q_{0}^{2}+|\mathbf{B}|^{2}\right. \\
& \left.-\left|\mathbf{B}_{0}\right|^{2}\right) \mathrm{d} x \\
A_{4} & =\int_{0}^{\tau} \int_{\Omega}\left(\mu_{\varepsilon} \nabla \mathbf{u}_{\epsilon}: \nabla \mathbf{v}+\nu_{\varepsilon} \nabla \mathbf{B}_{\varepsilon}: \nabla \mathbf{B}\right) \mathrm{d} x \mathrm{~d} t \\
A_{5} & =-\int_{0}^{\tau} \int_{\Omega} \varrho_{\epsilon} \mathbf{u}_{\epsilon} \cdot \partial_{t} \mathbf{v} \mathrm{d} x \mathrm{~d} t-\int_{0}^{\tau} \int_{\Omega}\left[\varrho_{\epsilon} \mathbf{u}_{\epsilon}\right. \\
& \left.\otimes \mathbf{u}_{\epsilon}: \nabla \mathbf{v}\right] \mathrm{d} x \mathrm{~d} t-\int_{0}^{\tau} \int_{\Omega} q_{\varepsilon} \partial_{t} q \mathrm{~d} x \mathrm{~d} t-\frac{1}{\varepsilon} \\
& \cdot \int_{0}^{\tau} \int_{\Omega} \varrho_{\epsilon} \nabla G \cdot \mathbf{v} \mathrm{d} x \mathrm{~d} t \\
A_{6} & =-\int_{0}^{\tau} \int_{\Omega}\left(\mathbf{B}_{\varepsilon} \cdot \nabla\right) \mathbf{B}_{\varepsilon} \cdot \mathbf{v}+\int_{0}^{\tau} \int_{\Omega} \mathbf{B}_{\varepsilon} \cdot[(\mathbf{v} \cdot \nabla) \mathbf{B} \\
& -(\mathbf{B} \cdot \nabla) \mathbf{v}] \mathrm{d} x \mathrm{~d} t \\
A_{7} & =\int_{0}^{\tau} \int_{\Omega}\left[\left(\mathrm{div} \mathbf{u}_{\epsilon}\right) \mathbf{B}_{\varepsilon}+\left(\mathbf{u}_{\epsilon} \cdot \nabla\right) \mathbf{B}_{\varepsilon}-\left(\mathbf{B}_{\varepsilon} \cdot \nabla\right) \mathbf{u}_{\epsilon}\right] \\
& -\mathbf{B} \mathrm{d} x \mathrm{~d} t .
\end{aligned}
$$

Step 3. From (19), it is easily seen that it shows that

$$
A_{1} \leq C \varepsilon
$$

Indeed, using (6), (19), and (21), we get

$$
\begin{aligned}
& \left\|\sqrt{\varrho_{0, \varepsilon}}\left(\mathbf{u}_{0, \varepsilon}-\nabla^{\perp} q_{0}\right)\right\|_{L^{2}(\Omega)}^{2} \\
& \leq C\left\|\sqrt{\varrho_{0, \varepsilon}} \mathbf{u}_{0, \varepsilon}-\nabla^{\perp} q_{0}\right\|_{L^{2}(\Omega)}^{2} \\
& \quad+C\left\|\left(\sqrt{\varrho_{0, \varepsilon}}-\sqrt{\widetilde{\varrho}_{\varepsilon}}\right) \nabla^{\perp} q_{0}\right\|_{L^{2}(\Omega)}^{2} \\
& \quad+C\left\|\left(\sqrt{\widetilde{\varrho}_{\varepsilon}}-1\right) \nabla^{\perp} q_{0}\right\|_{L^{2}(\Omega)}^{2} \leq C \varepsilon,
\end{aligned}
$$

where $\left\|\nabla^{\perp} q_{0}\right\|_{L^{\infty}(\Omega)} \leq C$ and the constant depends on the support of $G$, and we also have

$$
\begin{aligned}
\left\|H\left(q_{0, \varepsilon}\right)-q_{0}\right\|_{L^{2}(\Omega)}^{2} \leq & C\left\|H\left(q_{0, \varepsilon}\right)-q_{0, \varepsilon}\right\|_{L^{2}(\Omega)}^{2} \\
& +C\left\|q_{0, \varepsilon}-q_{0}\right\|_{L^{2}(\Omega)}^{2} \leq C \varepsilon,
\end{aligned}
$$

where we have used (21). Similarly, we get

$$
\begin{gathered}
\left\|q_{0}\left(H\left(q_{0, \varepsilon}\right)-q_{0}\right)\right\|_{L^{2}(\Omega)}^{2} \leq C \varepsilon, \\
\left\|\left(\varrho_{0, \varepsilon}-\widetilde{\varrho}_{\varepsilon}\right)\left|\nabla^{\perp} q_{0}\right|^{2}\right\|_{L^{2}(\Omega)}^{2} \leq C \varepsilon .
\end{gathered}
$$

By the regularity of $q$ in (11) and (34), we can estimate the first term of $A_{2}$ as follows:

$$
\begin{aligned}
& \int_{\Omega}\left(\varrho_{\epsilon}-\widetilde{\varrho}_{\varepsilon}\right)\left|\nabla^{\perp} q\right|^{2} \mathrm{~d} x \\
& \leq C\left\|\left(\varrho_{\epsilon}-\widetilde{\varrho}_{\varepsilon}\right) 1_{\left|\varrho_{\varepsilon}-\widetilde{\varrho}_{\varepsilon}\right| \leq 1 / 2}\right\|_{L^{\infty}\left(0, T ; L^{2}(\Omega)\right)} \\
& \quad+C\left\|\left(\varrho_{\epsilon}^{\gamma}+1\right) 1_{\left|\varrho_{\epsilon}-\widetilde{\varrho}_{\varepsilon}\right| \geq 1 / 2}\right\|_{L^{\infty}\left(0, T ; L^{2}(\Omega)\right)} \\
& \leq C\left(\varepsilon+\varepsilon^{2}\right),
\end{aligned}
$$

where the Sobolev imbedding theorem $H^{2} \subset L^{\infty}$ implies that $\nabla^{\perp} q \in L^{\infty}(\Omega)$. The estimate of the second term of $A_{2}$ is also given in [10] such that

$$
\int_{\Omega} q\left(H_{\varepsilon}\left(q_{\varepsilon}\right)-q_{\varepsilon}\right) \mathrm{d} x \leq C \varepsilon^{2 / \gamma} .
$$

To handle $A_{3}$, we multiply $q$ to system (9), which yields

$$
\begin{aligned}
& \frac{1}{2} \int_{\Omega}\left(\left|\nabla^{\perp} q\right|^{2}+q^{2}\right) \mathrm{d} x-\frac{1}{2} \int_{\Omega}\left(\left|\nabla^{\perp} q_{0}\right|^{2}+q_{0}^{2}\right) \mathrm{d} x \\
& \quad=\int_{0}^{\tau} \int_{\Omega}(\mathbf{B} \cdot \nabla) \mathbf{B} \cdot \mathbf{v} \mathrm{d} x \mathrm{~d} t,
\end{aligned}
$$

while

$$
\frac{1}{2} \int_{0}^{\tau} \int_{\Omega} \nabla^{\perp} q \cdot \nabla G q \mathrm{~d} x \mathrm{~d} t=0 .
$$

Multiplying B to the magnetic field equation, we obtain the following energy equation:

$$
\begin{aligned}
\frac{1}{2} \int_{\Omega}|\mathbf{B}|^{2} \mathrm{~d} x-\frac{1}{2} \int_{\Omega}\left|\mathbf{B}_{0}\right|^{2} \mathrm{~d} x \\
\quad=-\int_{0}^{\tau} \int_{\Omega}(\mathbf{B} \cdot \nabla) \mathbf{B} \cdot \mathbf{v} \mathrm{d} x \mathrm{~d} t .
\end{aligned}
$$

Adding (52) and (54), it follows that

$$
A_{3}=\frac{1}{2} \int_{\Omega}\left(\widetilde{\varrho}_{\varepsilon}-1\right)\left(\left|\nabla^{\perp} q\right|^{2}-\left|\nabla^{\perp} q_{0}\right|^{2}\right) \mathrm{d} x \leq C \varepsilon,
$$

where we have used (6). Let us next show that the viscosity term $A_{4}$ vanishes:

$$
\begin{aligned}
& \left|\int_{0}^{\tau} \int_{\Omega}\left(\mu_{\varepsilon} \nabla \mathbf{u}_{\epsilon}: \nabla \mathbf{v}+\nu_{\varepsilon} \nabla \mathbf{B}_{\varepsilon}: \nabla \mathbf{B}\right) \mathrm{d} x \mathrm{~d} t\right| \\
& \leq \frac{\mu_{\varepsilon}}{2} \int_{0}^{\tau} \int_{\Omega}\left|\nabla \mathbf{u}_{\epsilon}\right|^{2} \mathrm{~d} x \mathrm{~d} t+\frac{\nu_{\varepsilon}}{2} \int_{0}^{t} \int_{\Omega}\left|\nabla \mathbf{B}_{\varepsilon}\right|^{2} \mathrm{~d} x \mathrm{~d} t \\
& \quad+C\left(\mu_{\varepsilon}+\nu_{\varepsilon}\right),
\end{aligned}
$$

where we have here used (4) and (11). 
Step 4. For this part, we need to estimate the convective term of $A_{5}$ and the term can be expressed in the following form:

$$
\begin{gathered}
-\int_{0}^{\tau} \int_{\Omega} \varrho_{\epsilon}\left(\mathbf{u}_{\epsilon}-\mathbf{v}\right) \otimes\left(\mathbf{u}_{\epsilon}-\mathbf{v}\right): \nabla \mathbf{v} \mathrm{d} x \mathrm{~d} t \\
-\int_{0}^{\tau} \int_{\Omega} \varrho_{\epsilon} \mathbf{v} \otimes \mathbf{u}_{\epsilon}: \nabla \mathbf{v} \mathrm{d} x \mathrm{~d} t \\
-\int_{0}^{\tau} \int_{\Omega} \varrho_{\epsilon} \mathbf{u}_{\epsilon} \otimes \mathbf{v}: \nabla \mathbf{v} \mathrm{d} x \mathrm{~d} t \\
+\int_{0}^{\tau} \int_{\Omega} \varrho_{\epsilon} \mathbf{v} \otimes \mathbf{v}: \nabla \mathbf{v} \mathrm{d} x \mathrm{~d} t:=\sum_{j=1}^{4} I_{j} .
\end{gathered}
$$

The first term $I_{1}$ can be controlled by

$$
I_{1} \leq C \int_{0}^{\tau} \mathscr{E}_{\varepsilon}(t) \mathrm{d} t
$$

where, by (11), $\nabla \mathbf{v} \in H^{2}(\Omega)$ together with the Sobolev embedding, which implies that

$$
\|\nabla \mathbf{v}\|_{L^{\infty}(\Omega)} \leq C
$$

$I_{2}$ is reformulated by

$$
I_{2}=-\int_{0}^{\tau} \int_{\Omega} \varrho_{\epsilon} \mathbf{u}_{\epsilon} \cdot((\mathbf{v} \cdot \nabla) \mathbf{v}) \mathrm{d} x \mathrm{~d} t
$$

For $I_{3}$, employing the estimates of (32) and (34) together with the continuity equation (1), we get

$$
\begin{aligned}
I_{3}= & -\frac{1}{2} \int_{0}^{\tau} \int_{\Omega} \varrho_{\epsilon} \mathbf{u}_{\epsilon} \cdot \nabla|\mathbf{v}|^{2} \mathrm{~d} x \mathrm{~d} t \\
= & \frac{1}{2} \int_{0}^{\tau} \int_{\Omega}\left(\rho_{\epsilon}-\tilde{\rho}_{\varepsilon}\right) \partial_{t}|\mathbf{v}|^{2} \mathrm{~d} x \mathrm{~d} t \\
& -\frac{1}{2} \int_{\Omega}\left(\varrho_{\epsilon}-\widetilde{\varrho}_{\varepsilon}\right)|\mathbf{v}(\tau)|^{2} \mathrm{~d} x \\
& +\frac{1}{2} \int_{\Omega}\left(\varrho_{0, \varepsilon}-\widetilde{\varrho}_{\varepsilon}\right)\left|\mathbf{v}_{0}\right|^{2} \mathrm{~d} x \leq C\left(\varepsilon+\varepsilon^{2}\right) .
\end{aligned}
$$

To handle $I_{4}$, we use $\operatorname{div} \mathbf{v}=0$ to obtain

$$
\begin{aligned}
I_{4}= & -\int_{0}^{\tau} \int_{\Omega}\left(\varrho_{\epsilon}-\widetilde{\varrho}_{\varepsilon}\right)(\mathbf{v} \cdot \nabla) \mathbf{v} \cdot \mathbf{v} \mathrm{d} x \mathrm{~d} t \\
& -\int_{0}^{\tau} \int_{\Omega}\left(\widetilde{\varrho}_{\varepsilon}-1\right)(\mathbf{v} \cdot \nabla \mathbf{v}) \cdot \mathbf{v} \mathrm{d} x \mathrm{~d} t \leq C\left(\varepsilon+\varepsilon^{2}\right),
\end{aligned}
$$

where we have used (32) and (33). Thus, $A_{5}$ is written by

$$
\begin{aligned}
A_{5} & \leq-\int_{0}^{\tau} \int_{\Omega}\left(\varrho_{\epsilon} \mathbf{u}_{\epsilon} \cdot\left(\partial_{t} \mathbf{v}+(\mathbf{v} \cdot \nabla) \mathbf{v}\right)+q_{\varepsilon} \nabla G \cdot \mathbf{v}\right. \\
& \left.+q_{\varepsilon} \partial_{t} q\right) \mathrm{d} x \mathrm{~d} t+C \int_{0}^{\tau} \mathscr{E}_{\varepsilon}(t) \mathrm{d} t+C(\varepsilon \\
& \left.+\varepsilon^{2}\right)
\end{aligned}
$$

where

$$
\begin{aligned}
& -\frac{1}{\varepsilon} \int_{0}^{\tau} \int_{\Omega} \varrho_{\epsilon} \nabla G \cdot \mathbf{v} \mathrm{d} x \mathrm{~d} t \\
& =-\int_{0}^{\tau} \int_{\Omega} \frac{\widetilde{\varrho}_{\varepsilon}}{\varepsilon} \nabla G \cdot \mathbf{v} \mathrm{d} x \mathrm{~d} t+\int_{0}^{\tau} \int_{\Omega} q_{\varepsilon} \nabla G \cdot \mathbf{v} \mathrm{d} x \mathrm{~d} t \\
& \quad=\int_{0}^{\tau} \int_{\Omega} q_{\varepsilon} \nabla G \cdot \mathbf{v} \mathrm{d} x \mathrm{~d} t
\end{aligned}
$$

together with using (5) and $\operatorname{div} \mathbf{v}=0$. To handle (63), we need the following lemma.

Lemma 4. Let $\left(\varrho_{\epsilon}, \mathbf{u}_{\epsilon}, \mathbf{B}_{\varepsilon}\right)$ be global weak solutions of (1)-(3) in the sense of Definition 2. Then, one has the following inequality:

$$
\left|\int_{0}^{\tau} \int_{\Omega}\left(\varrho_{\epsilon} \mathbf{u}_{\epsilon}-\nabla^{\perp} q_{\varepsilon}\right) \cdot \psi \mathrm{d} x \mathrm{~d} t\right| \leq C \varepsilon
$$

for any test function $\psi$.

Proof. We use the test function $\psi^{\perp}$ to the moment equation (2) to deduce

$$
\begin{aligned}
& \int_{0}^{\tau} \int_{\Omega}\left(\varrho_{\epsilon} \mathbf{u}_{\epsilon}-\nabla^{\perp} q_{\varepsilon}\right) \cdot \psi \mathrm{d} x \mathrm{~d} t=\varepsilon \int_{\Omega}\left(\varrho_{\epsilon} \mathbf{u}_{\epsilon}\right)(\tau) \\
& \cdot \psi^{\perp}(\tau) \mathrm{d} x-\varepsilon \int_{\Omega}\left(\varrho_{0, \varepsilon} \mathbf{u}_{0, \varepsilon}\right) \cdot \psi_{0}^{\perp} \mathrm{d} x \\
& +\varepsilon \int_{0}^{\tau} \int_{\Omega}\left(\varrho_{\epsilon} \mathbf{u}_{\epsilon}\right) \cdot \partial_{t} \psi^{\perp} \mathrm{d} x \mathrm{~d} t \\
& +\varepsilon \int_{0}^{t} \int_{\Omega}\left(\varrho_{\epsilon} \mathbf{u}_{\epsilon} \otimes \mathbf{u}_{\epsilon}\right): \nabla \psi^{\perp} \mathrm{d} x \mathrm{~d} t \\
& +\varepsilon \int_{0}^{\tau} \int_{\Omega} \frac{1}{\varepsilon^{2} \gamma}\left(\varrho_{\epsilon}^{\gamma}-\widetilde{\varrho}_{\varepsilon}^{\gamma}-\gamma \widetilde{\varrho}_{\varepsilon}^{\gamma-1}\left(\varrho_{\epsilon}-\widetilde{\varrho}_{\varepsilon}\right)\right)
\end{aligned}
$$

$$
\begin{aligned}
& \cdot \operatorname{div} \psi^{\perp} \mathrm{d} x \mathrm{~d} t-\varepsilon \int_{0}^{\tau} \int_{\Omega} \frac{\varrho_{\epsilon}-\tilde{\varrho}_{\varepsilon}}{\varepsilon} \nabla G \cdot \psi^{\perp} \mathrm{d} x \mathrm{~d} t \\
& -\varepsilon \mu_{\varepsilon} \int_{0}^{\tau} \int_{\Omega} \nabla \mathbf{u}_{\epsilon}: \nabla \psi^{\perp} \mathrm{d} x \mathrm{~d} t-\varepsilon\left(\mu_{\varepsilon}+\lambda_{\varepsilon}\right) \\
& \cdot \int_{0}^{t} \int_{\Omega} \operatorname{div} \mathbf{u}_{\epsilon} \operatorname{div} \psi^{\perp} \mathrm{d} x \mathrm{~d} t+\varepsilon \int_{0}^{\tau} \int_{\Omega}\left(\mathbf{B}_{\varepsilon} \cdot \nabla\right) \mathbf{B}_{\varepsilon} \\
& \cdot \psi^{\perp}+\frac{\varepsilon}{2} \int_{0}^{t} \int_{\Omega}\left|\mathbf{B}_{\varepsilon}\right|^{2} \operatorname{div} \psi^{\perp} \mathrm{d} x \mathrm{~d} t .
\end{aligned}
$$

In virtue of the estimates in (19)-(21) and (24)-(27), the terms of the right-hand side in (66) can be controlled and so it proved (65). 
Making use of Lemma 4 together with $\mathbf{v}=\nabla^{\perp} q$ and $\psi=$ $\partial_{t} \nabla^{\perp} q+\left(\nabla^{\perp} q \cdot \nabla\right) \nabla^{\perp} q$ and integrating by parts, we obtain that

$$
\begin{aligned}
A_{5} & =-\int_{0}^{\tau} \int_{\Omega} \varrho_{\epsilon} \mathbf{u}_{\epsilon} \cdot\left(\partial_{t} \nabla^{\perp} q+\left(\nabla^{\perp} q \cdot \nabla\right) \nabla^{\perp} q\right) \mathrm{d} x \mathrm{~d} t \\
& -\int_{0}^{\tau} \int_{\Omega} q_{\varepsilon}\left(\partial_{t} q+\nabla G \cdot \nabla^{\perp} q\right) \mathrm{d} x \mathrm{~d} t \\
& +C \int_{0}^{\tau} \mathscr{E}_{\varepsilon}(t) \mathrm{d} t+C\left(\varepsilon+\varepsilon^{2}\right) \\
& \leq \int_{0}^{\tau} \int_{\Omega} q_{\varepsilon}\left[\partial_{t}(\Delta q-q)+\left(\nabla^{\perp} q \cdot \nabla\right) \Delta q+\nabla G\right. \\
& \left.\cdot \nabla^{\perp} q\right] \mathrm{d} x \mathrm{~d} t+C \int_{0}^{\tau} \mathscr{E}_{\varepsilon}(t) \mathrm{d} t+C\left(\varepsilon+\varepsilon^{2}\right) \\
& =\int_{0}^{\tau} \int_{\Omega} q_{\varepsilon} \operatorname{div}{ }^{\perp}\left[(\mathbf{B} \cdot \nabla) \mathbf{B}-\frac{1}{2} \nabla|\mathbf{B}|^{2}\right] \mathrm{d} x \mathrm{~d} t \\
& +C \int_{0}^{\tau} \mathscr{E}_{\varepsilon}(t) \mathrm{d} t+C\left(\varepsilon+\varepsilon^{2}\right) \leq-\int_{0}^{\tau} \int_{\Omega} \varrho_{\epsilon} u_{\epsilon} \\
& \cdot\left[(\mathbf{B} \cdot \nabla) \mathbf{B}-\frac{1}{2} \nabla|\mathbf{B}|^{2}\right] \mathrm{d} x \mathrm{~d} t \\
& +C \int_{0}^{\tau} \mathscr{E}_{\varepsilon}(t) \mathrm{d} t+C\left(\varepsilon+\varepsilon^{2}\right),
\end{aligned}
$$

where we have used $\psi=(\mathbf{B} \cdot \nabla) \mathbf{B}-(1 / 2) \nabla|\mathbf{B}|^{2}$ as a test function on the last line of (67). Thus, the relative entropy gives

$$
\begin{aligned}
& \mathscr{E}_{\varepsilon}(\tau)+\frac{1}{2} \int_{0}^{\tau} \int_{\Omega}\left(\mu_{\varepsilon}\left|\nabla \mathbf{u}_{\epsilon}\right|^{2}+\left(\mu_{\varepsilon}+\lambda_{\varepsilon}\right)\left|\operatorname{div} \mathbf{u}_{\epsilon}\right|^{2}\right. \\
& \left.+v_{\varepsilon}\left|\nabla \mathbf{B}_{\varepsilon}\right|^{2}\right) \mathrm{d} x \mathrm{~d} t \leq-\int_{0}^{\tau} \int_{\Omega}\left(\mathbf{B}_{\varepsilon} \cdot \nabla\right) \mathbf{B}_{\varepsilon} \\
& \cdot \mathbf{v}+\int_{0}^{\tau} \int_{\Omega} \mathbf{B}_{\varepsilon} \cdot[(\mathbf{v} \cdot \nabla) \mathbf{B}-(\mathbf{B} \cdot \nabla) \mathbf{v}] \mathrm{d} x \mathrm{~d} t \\
& +\int_{0}^{\tau} \int_{\Omega}\left[\left(\operatorname{div} \mathbf{u}_{\epsilon}\right) \mathbf{B}_{\varepsilon}+\left(\mathbf{u}_{\epsilon} \cdot \nabla\right) \mathbf{B}_{\varepsilon}-\left(\mathbf{B}_{\varepsilon} \cdot \nabla\right) \mathbf{u}_{\epsilon}\right] \\
& \cdot \mathbf{B} \mathrm{d} x \mathrm{~d} t-\int_{0}^{\tau} \int_{\Omega} \varrho_{\epsilon} \mathbf{u}_{\epsilon} \cdot[(\mathbf{B} \cdot \nabla) \mathbf{B} \\
& \left.-\frac{1}{2} \nabla|\mathbf{B}|^{2}\right] \mathrm{d} x \mathrm{~d} t+\int_{0}^{\tau} \mathscr{E}_{\varepsilon}(t) \mathrm{d} t+C(\varepsilon \\
& \left.+\varepsilon^{2 / \gamma}+\varepsilon^{2}+\varepsilon^{\theta}+\varepsilon^{\sigma}\right) .
\end{aligned}
$$

Step 5. Finally, we now handle the part of magnetic field. We apply the integration by parts and the facts $\operatorname{div} \mathbf{B}_{\varepsilon}=0, \operatorname{div} \mathbf{B}=$ 0 , and $\operatorname{div} \mathbf{v}=0$ to obtain

$$
\begin{gathered}
\mathscr{E}_{\varepsilon}(t)+\frac{1}{2} \int_{0}^{\tau} \int_{\Omega} \mu_{\varepsilon}\left|\nabla_{x} \mathbf{u}_{\epsilon}\right|^{2}+\left(\mu_{\varepsilon}+\lambda_{\varepsilon}\right)\left|\operatorname{div} \mathbf{u}_{\epsilon}\right|^{2} \\
+v_{\varepsilon}\left|\nabla \mathbf{B}_{\varepsilon}\right|^{2} \mathrm{~d} x \mathrm{~d} t \leq \int_{0}^{\tau} \int_{\Omega}\left(\widetilde{\varrho}_{\varepsilon}-\varrho_{\epsilon}\right) \mathbf{u}_{\epsilon} \\
\cdot(\mathbf{B} \cdot \nabla) \mathbf{B} \mathrm{d} x \mathrm{~d} t+\frac{1}{2} \int_{0}^{\tau} \int_{\Omega}\left(\widetilde{\varrho}_{\varepsilon}-\varrho_{\epsilon}\right) \mathbf{u}_{\epsilon}
\end{gathered}
$$

$$
\begin{aligned}
& \cdot \nabla|\mathbf{B}|^{2} \mathrm{~d} x \mathrm{~d} t+\int_{0}^{\tau} \int_{\Omega}\left(1-\widetilde{\varrho}_{\varepsilon}\right) \mathbf{u}_{\epsilon} \\
& \cdot(\mathbf{B} \cdot \nabla) \mathbf{B} \mathrm{d} x \mathrm{~d} t+\frac{1}{2} \int_{0}^{\tau} \int_{\Omega}\left(1-\widetilde{\varrho}_{\varepsilon}\right) \mathbf{u}_{\epsilon} \\
& \cdot \nabla|\mathbf{B}|^{2} \mathrm{~d} x \mathrm{~d} t+\int_{0}^{\tau} \int_{\Omega}\left(\mathbf{B}_{\varepsilon}-\mathbf{B}\right) \cdot \nabla \mathbf{v} \\
& \cdot\left(\mathbf{B}_{\varepsilon}-\mathbf{B}\right) \mathrm{d} x \mathrm{~d} t+\int_{0}^{\tau} \int_{\Omega}\left(\mathbf{B}_{\varepsilon}-\mathbf{B}\right) \cdot \nabla \mathbf{B} \\
& \cdot\left(\mathbf{u}_{\epsilon}-\mathbf{v}\right) \mathrm{d} x \mathrm{~d} t-\int_{0}^{\tau} \int_{\Omega}\left(\mathbf{u}_{\epsilon}-\mathbf{v}\right) \cdot \nabla \mathbf{B} \\
& \cdot\left(\mathbf{B}_{\varepsilon}-\mathbf{B}\right) \mathrm{d} x \mathrm{~d} t+C \int_{0}^{\tau} \mathscr{E}_{\varepsilon}(t) \mathrm{d} t+C(\varepsilon \\
& \left.+\varepsilon^{2 / \gamma}+\varepsilon^{2}+\varepsilon^{\theta}+\varepsilon^{\sigma}\right):=\sum_{j=1}^{7} D_{j}+C \int_{0}^{\tau} \mathscr{E}_{\varepsilon}(t) \mathrm{d} t \\
& +C\left(\varepsilon+\varepsilon^{2 / \gamma}+\varepsilon^{2}+\varepsilon^{\theta}+\varepsilon^{\sigma}\right) .
\end{aligned}
$$

For the term $D_{6}$, making use of (32), (33), and (37), together with the Sobolev embedding and Holder's inequality, it follows that

$$
\begin{aligned}
& \int_{0}^{\tau} \int_{\Omega}\left(\mathbf{B}_{\varepsilon}-\mathbf{B}\right) \cdot \nabla \mathbf{B} \cdot\left(\mathbf{u}_{\epsilon}-\mathbf{v}\right) \mathrm{d} x \mathrm{~d} t \\
& =\int_{0}^{\tau} \int_{\Omega}\left(\mathbf{B}_{\varepsilon}-\mathbf{B}\right) \cdot \nabla \mathbf{B} \cdot \sqrt{\varrho_{\epsilon}}\left(\mathbf{u}_{\epsilon}-\mathbf{v}\right) \mathrm{d} x \mathrm{~d} t \\
& +\int_{0}^{\tau} \int_{\Omega}\left(\sqrt{\widetilde{\varrho}_{\varepsilon}}-\sqrt{\varrho_{\epsilon}}\right)\left(\mathbf{B}_{\varepsilon}-\mathbf{B}\right) \cdot \nabla \mathbf{B} \\
& \cdot\left(\mathbf{u}_{\epsilon}-\mathbf{v}\right) \mathrm{d} x \mathrm{~d} t+\int_{0}^{\tau} \int_{\Omega}\left(1-\sqrt{\widetilde{\varrho}_{\varepsilon}}\right)\left(\mathbf{B}_{\varepsilon}-\mathbf{B}\right) \\
& \cdot \nabla \mathbf{B} \cdot\left(\mathbf{u}_{\epsilon}-\mathbf{v}\right) \mathrm{d} x \mathrm{~d} t \leq C \| \sqrt{\varrho_{\epsilon}} \\
& -\sqrt{\widetilde{\varrho}_{\varepsilon}}\left\|_{L^{\infty}\left(0, T ; L^{2}(\Omega)\right)}\right\| \mathbf{B}_{\varepsilon}\left\|_{L^{2}\left(0, T ; L^{6}(\Omega)\right)}\right\| \mathbf{u}_{\epsilon} \|_{L^{2}\left(0, T ; L^{3}(\Omega)\right)} \\
& +C\left\|\sqrt{\varrho_{\epsilon}}-\sqrt{\widetilde{\varrho_{\varepsilon}}}\right\|_{L^{\infty}\left(0, T ; L^{2}(\Omega)\right)}\left(\left\|\mathbf{u}_{\epsilon}\right\|_{L^{2}\left(0, T ; L^{2}(\Omega)\right)}\right. \\
& \left.+\left\|\mathbf{B}_{\varepsilon}\right\|_{L^{2}\left(0, T ; L^{2}(\Omega)\right)}+1\right)+C\left\|1-\sqrt{\widetilde{\varrho}_{\varepsilon}}\right\|_{L^{\infty}\left(0, T ; L^{2}(\Omega)\right)} \\
& \cdot\left\|\mathbf{B}_{\varepsilon}\right\|_{L^{2}\left(0, T ; L^{6}(\Omega)\right)}\left\|\mathbf{u}_{\epsilon}\right\|_{L^{2}\left(0, T ; L^{3}(\Omega)\right)}+C \| 1 \\
& -\sqrt{\widetilde{\varrho}_{\varepsilon}} \|_{L^{\infty}\left(0, T ; L^{2}(\Omega)\right)}\left(\left\|\mathbf{u}_{\epsilon}\right\|_{L^{2}\left(0, T ; L^{2}(\Omega)\right)}\right. \\
& \left.+\left\|\mathbf{B}_{\varepsilon}\right\|_{L^{2}\left(0, T ; L^{2}(\Omega)\right)}+1\right)+C \int_{0}^{\tau} \mathscr{E}_{\varepsilon}(t) \mathrm{d} t \\
& \leq C\left(\varepsilon^{1-(\theta+\sigma) / 2}+\varepsilon+\varepsilon^{5 / 3-\theta / 2}\right)+C \int_{0}^{\tau} \mathscr{E}_{\varepsilon}(t) \mathrm{d} t,
\end{aligned}
$$


where we have used the assumption of viscosity $0<\theta+\sigma<2$, (6), and the Sobolev embedding theorem:

$$
\begin{aligned}
&\left\|\mathbf{B}_{\varepsilon}\right\|_{L^{2}\left(0, T ; L^{6}(\Omega)\right)} \leq C\left\|\nabla \mathbf{B}_{\varepsilon}\right\|_{L^{2}\left(0, T ; L^{2}(\Omega)\right)}, \\
&\left\|\mathbf{u}_{\epsilon}\right\|_{L^{2}\left(0, T ; L^{3}(\Omega)\right)} \leq C\left\|\nabla \mathbf{u}_{\epsilon}\right\|_{L^{2}\left(0, T ; L^{2}(\Omega)\right)} .
\end{aligned}
$$

Similarly, the term $D_{7}$ can also be controlled by

$$
D_{7} \leq C \int_{0}^{\tau} \mathscr{E}_{\varepsilon}(t) \mathrm{d} t+C\left(\varepsilon^{1-(\theta+\sigma) / 2}+\varepsilon+\varepsilon^{5 / 3-\theta / 2}\right) .
$$

Finally, we estimate $D_{2}, D_{3}, D_{4}$, and $D_{5}$ with the same method. We now rewrite $D_{1}$ as follows:

$$
\begin{aligned}
D_{1}= & \int_{0}^{\tau} \int_{\Omega}\left(\widetilde{\varrho}_{\varepsilon}-\varrho_{\epsilon}\right) \mathbf{u}_{\epsilon} \cdot(\mathbf{B} \cdot \nabla) \mathbf{B} \mathrm{d} x \mathrm{~d} t \\
= & \int_{0}^{\tau} \int_{\Omega} \sqrt{\widetilde{\varrho}_{\varepsilon}}\left(\sqrt{\widetilde{\varrho}_{\varepsilon}}-\sqrt{\varrho_{\epsilon}}\right) \mathbf{u}_{\epsilon} \cdot(\mathbf{B} \cdot \nabla) \mathbf{B} \mathrm{d} x \mathrm{~d} t \\
& +\int_{0}^{\tau} \int_{\Omega}\left(\sqrt{\widetilde{\varrho}_{\varepsilon}}-\sqrt{\varrho_{\epsilon}}\right) \sqrt{\varrho_{\epsilon}} \mathbf{u}_{\epsilon} \cdot(\mathbf{B} \cdot \nabla) \mathbf{B} \mathrm{d} x \mathrm{~d} t .
\end{aligned}
$$

Thus, from the estimates of (32), (33), and (37), together with the estimate of (24), we get

$$
D_{1} \leq C\left(\varepsilon+\varepsilon^{5 / 3-\theta / 2}\right),
$$

where we have again used the assumption of viscosity $0<$ $\theta+\sigma<2$ and so

$$
\sum_{j=1}^{4} D_{j} \leq C\left(\varepsilon+\varepsilon^{5 / 3-\theta / 2}\right) .
$$

Consequently, the relative entropy in (69) is given by

$$
\begin{aligned}
& \mathscr{E}_{\varepsilon}(\tau)+\frac{1}{2} \int_{0}^{\tau} \int_{\Omega} \mu_{\varepsilon}\left|\nabla_{x} \mathbf{u}_{\epsilon}\right|^{2}+\left(\mu_{\varepsilon}+\lambda_{\varepsilon}\right)\left|\operatorname{div} \mathbf{u}_{\epsilon}\right|^{2} \\
& \quad+\nu_{\varepsilon}\left|\nabla \mathbf{B}_{\varepsilon}\right|^{2} \mathrm{~d} x \mathrm{~d} t \leq C \int_{0}^{\tau} \mathscr{C}_{\varepsilon}(t) \mathrm{d} t \\
& \quad+C\left(\varepsilon^{1-(\theta+\sigma) / 2}+\varepsilon+\varepsilon^{2 / \gamma}+\varepsilon^{2}+\varepsilon^{5 / 3-\theta / 2}+\varepsilon^{\theta}+\varepsilon^{\sigma}\right) .
\end{aligned}
$$

Step 6. Let us apply Grönwall's inequality to (76) in order to obtain

$$
\begin{aligned}
\mathscr{E}_{\varepsilon} & (\tau) \\
& \leq C\left(\varepsilon^{1-(\theta+\sigma) / 2}+\varepsilon+\varepsilon^{2 / \gamma}+\varepsilon^{2}+\varepsilon^{5 / 3-\theta / 2}+\varepsilon^{\theta}+\varepsilon^{\sigma}\right) \\
& \leq C \varepsilon^{\kappa}
\end{aligned}
$$

for any $\tau \in[0, T]$, where the number $\kappa$ is defined in (23). Note that

$$
\begin{aligned}
\int_{K}\left|q_{\varepsilon}-q\right| \mathrm{d} x \leq & \int_{\Omega}\left|q_{\varepsilon}-H_{\varepsilon}\left(q_{\varepsilon}\right)\right| \mathrm{d} x \\
& +C \int_{K}\left|H_{\varepsilon}\left(q_{\varepsilon}\right)-q\right|^{2} \mathrm{~d} x \\
\leq & C \int_{0}^{T} \mathscr{E}_{\varepsilon}(t) \mathrm{d} t+C \varepsilon^{\kappa},
\end{aligned}
$$

for any compact subset $K \subset \mathbb{R}^{2}$ and

$$
\begin{aligned}
& \int_{\Omega}\left|\sqrt{\varrho_{\epsilon}} \mathbf{u}_{\epsilon}-\nabla^{\perp} q\right|^{2} \mathrm{~d} x \\
& \leq \int_{\Omega}\left|\sqrt{\varrho_{\epsilon}} \mathbf{u}_{\epsilon}-\sqrt{\varrho_{\epsilon}} \nabla^{\perp} q+\left(\sqrt{\varrho_{\epsilon}}-\sqrt{\widetilde{\varrho}_{\varepsilon}}\right) \nabla^{\perp} q\right|^{2} \mathrm{~d} x \\
& \quad+C \int_{\Omega}\left|\left(\sqrt{\widetilde{\varrho}_{\varepsilon}}-1\right) \nabla^{\perp} q\right|^{2} \mathrm{~d} x \\
& \leq \int_{0}^{T} \mathscr{C}_{\varepsilon}(t) \mathrm{d} t+C \varepsilon^{\kappa},
\end{aligned}
$$

where $T$ is the given time in Theorem 1 . For the magnetic field, we obtain

$$
\int_{\Omega}\left|\mathbf{B}_{\varepsilon}-\mathbf{B}\right|^{2} \mathrm{~d} x \leq \int_{0}^{T} \mathscr{E}_{\varepsilon}(t) \mathrm{d} t .
$$

Using (77), (78), (79), and (80) and passing to the limit for $\varepsilon \rightarrow 0$, we prove (22). In conclusion, we get the target equation (9) by passing to the limits as $\varepsilon \rightarrow 0$, but it is sufficient to show that, for any test function $\phi$,

$$
\begin{array}{r}
\lim _{\varepsilon \rightarrow 0} \int_{0}^{T} \int_{\Omega} \frac{1}{\varepsilon} \varrho_{\epsilon} \nabla G \cdot \nabla^{\perp} \phi \mathrm{d} x \mathrm{~d} t \\
=\int_{0}^{T} \int_{\Omega} q \nabla G \cdot \nabla^{\perp} \phi \mathrm{d} x \mathrm{~d} t .
\end{array}
$$

Indeed,

$$
\begin{aligned}
\lim _{\varepsilon \rightarrow 0} \int_{0}^{T} \int_{\Omega} \frac{1}{\varepsilon} \varrho_{\varepsilon} \nabla G \cdot \nabla^{\perp} \phi \mathrm{d} x \mathrm{~d} t \\
=\lim _{\varepsilon \rightarrow 0} \int_{0}^{T} \int_{\Omega} \frac{1}{\varepsilon}\left(\widetilde{\varrho}_{\varepsilon}+\varepsilon q_{\varepsilon}\right) \nabla G \cdot \nabla^{\perp} \phi \mathrm{d} x \mathrm{~d} t \\
=\lim _{\varepsilon \rightarrow 0} \int_{0}^{T} \int_{\Omega} \frac{1}{\gamma \varepsilon^{2}} \nabla \widetilde{\varrho}_{\varepsilon}^{\gamma} \cdot \nabla^{\perp} \phi \mathrm{d} x \mathrm{~d} t \\
\quad+\lim _{\varepsilon \rightarrow 0} \int_{0}^{T} \int_{\Omega} q_{\varepsilon} \nabla G \cdot \nabla^{\perp} \phi \mathrm{d} x \mathrm{~d} t \\
=\int_{0}^{T} \int_{\Omega} q \nabla G \cdot \nabla^{\perp} \phi \mathrm{d} x \mathrm{~d} t,
\end{aligned}
$$

where we have used $\operatorname{div} \nabla^{\perp}=0$ and (5).

\section{Conflicts of Interest}

The author declares that there are no conflicts of interest regarding the publication of this paper.

\section{Acknowledgments}

The work of Young-Sam Kwon was supported by the research fund of Dong-A University. 


\section{References}

[1] X. Hu and D. Wang, "Global existence and large-time behavior of solutions to the three-dimensional equations of compressible magnetohydrodynamic flows," Archive for Rational Mechanics and Analysis, vol. 197, no. 1, pp. 203-238, 2010.

[2] N. Masmoudi, "Incompressible, inviscid limit of the compressible Navier-Stokes system," Ann. Inst. H.Poincaré Anal. NonLinéaire, vol. 18, no. 2, pp. 199-224, 2001.

[3] S. Jiang, Q. Ju, and F. Li, "Incompressible limit of the compressible magnetohydrodynamic equations with periodic boundary conditions," Communications in Mathematical Physics, vol. 297, no. 2, pp. 371-400, 2010.

[4] S. Jiang, Q. Ju, and F. Li, "Incompressible limit of the compressible magnetohydrodynamic equations with vanishing viscosity coefficients," SIAM Journal on Mathematical Analysis, vol. 42, no. 6, pp. 2539-2553, 2010.

[5] E. Feireisl and A. n. Novotný, "Inviscid incompressible limits of the full Navier-Stokes-Fourier system," Communications in Mathematical Physics, vol. 321, no. 3, pp. 605-628, 2013.

[6] E. Feireisl and A. n. Novotný, "Inviscid incompressible limits under mild stratification: a rigorous derivation of the EulerBoussinesq system," Applied Mathematics and Optimization, vol. 70, no. 2, pp. 279-307, 2014.

[7] M. Oliver, "Classical solutions for a generalized Euler equation in two dimensions," Journal of Mathematical Analysis and Applications, vol. 215, no. 2, pp. 471-484, 1997.

[8] P.-L. Lions and N. Masmoudi, "Incompressible limit for a viscous compressible fluid," J. Math.Pures Appl, vol. 215, pp. 585627, 1998.

[9] B. Desjardins and E. Grenier, "Low Mach number limit of viscous compressible flows in the whole space," The Royal Society of London. Proceedings. Series A. Mathematical, Physical and Engineering Sciences, vol. 455, no. 1986, pp. 2271-2279, 1999.

[10] A. Jungel, C. K. Lin, and K. C. Wu, "An asymptotic limit of a navier-stokes system with capillary effects," Communications in Mathematical Physics, vol. 329, no. 2, pp. 725-744, 2014. 


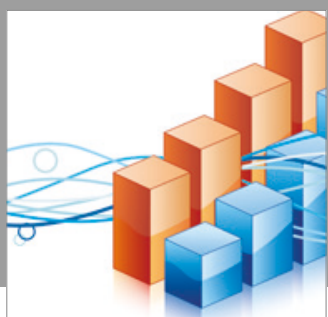

Advances in

Operations Research

vatersals

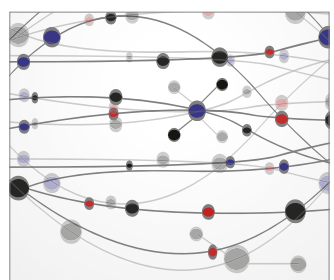

\section{The Scientific} World Journal
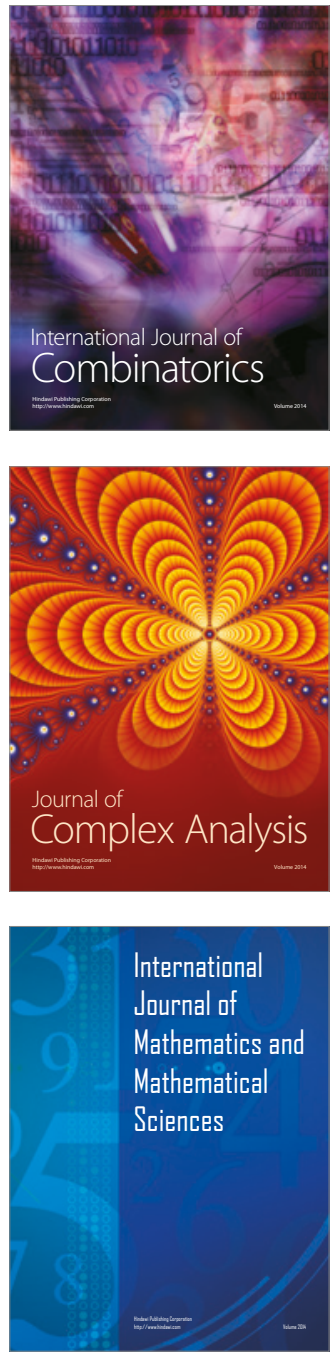
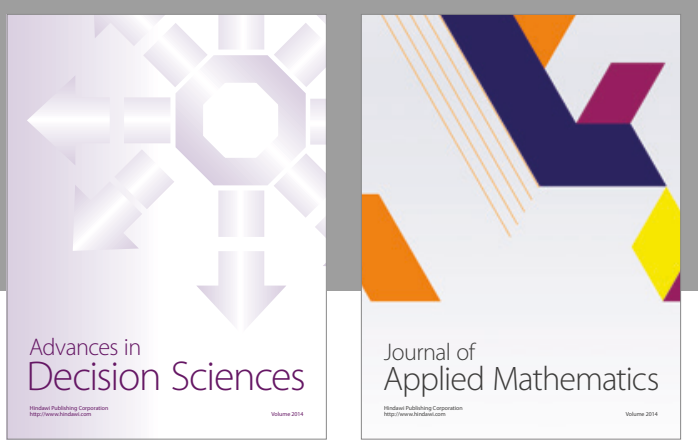

Algebra

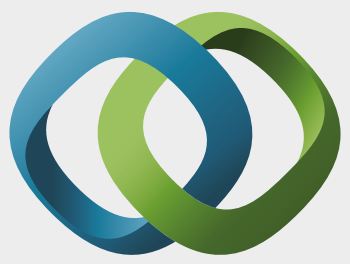

\section{Hindawi}

Submit your manuscripts at

https://www.hindawi.com
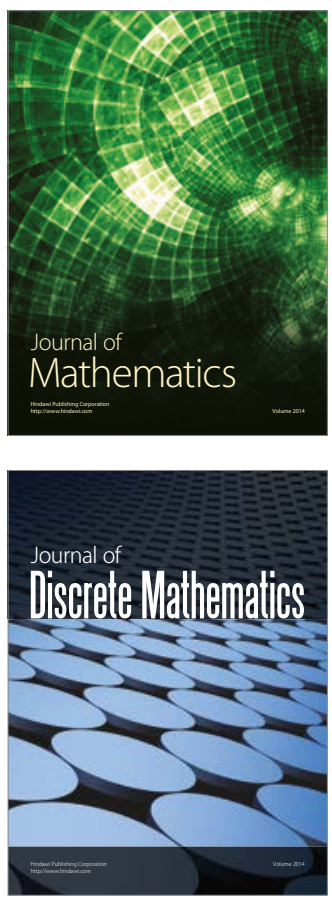

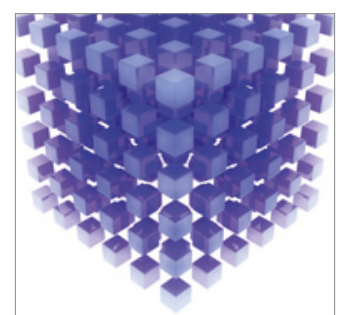

Mathematical Problems in Engineering
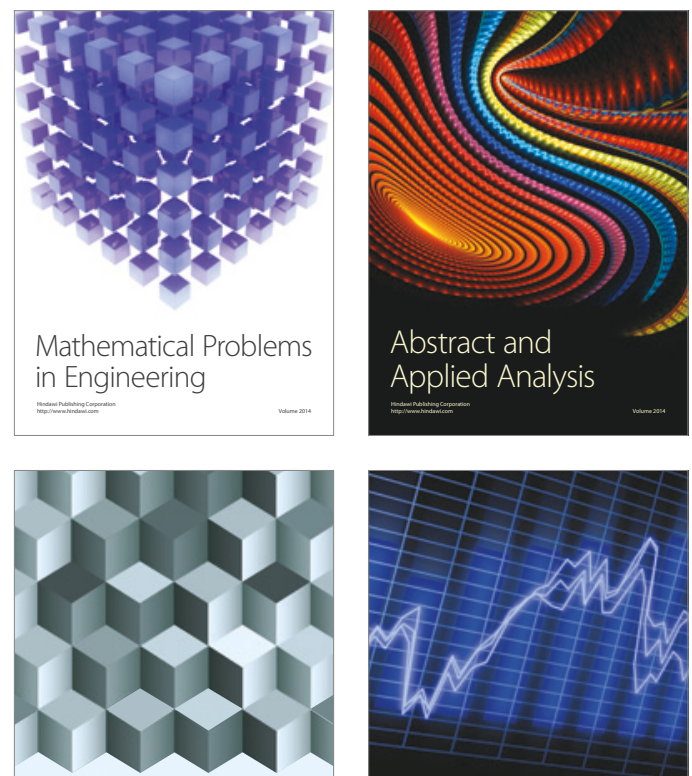

Journal of

Function Spaces

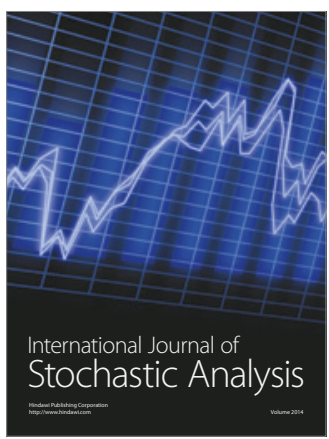

Probability and Statistics
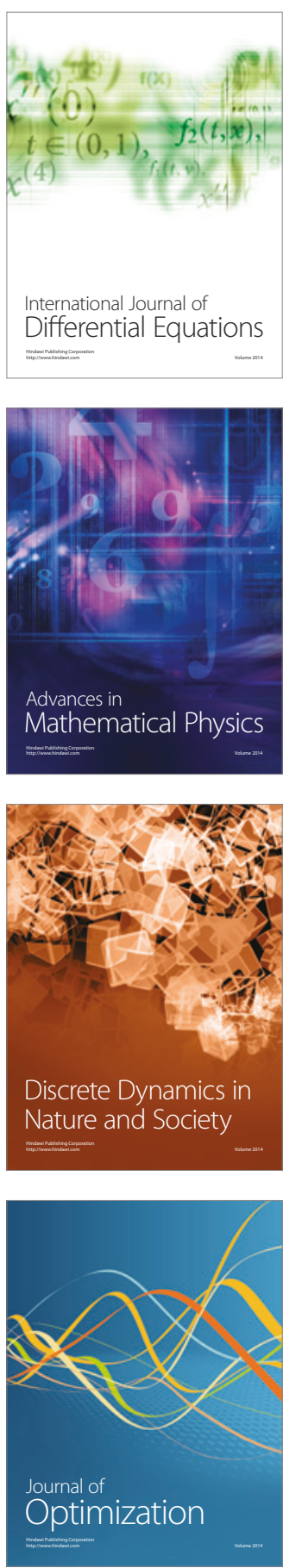